\title{
Faire confiance
}

\section{Daniel Schröpfer}

Dr méd., directeur du service médical de la ville de Zurich et membre du comité de I'Association des médecins dirigeants d'hôpitaux de Suisse (AMDHS),

membre de la rédaction

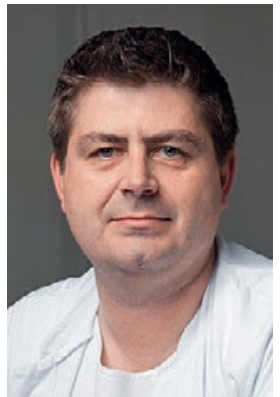

Depuis plusieurs années, j'exerce la médecine de premier recours auprès de personnes vulnérables. Je m'occupe notamment de personnes qui suivent un traitement de substitution, demandent l'asile, sont sans papiers ou n'ont pas d'assurance-maladie. Leur prise en charge est garantie, tant sur le plan médical que psychiatrique, mais contrairement aux patientes et patients dont je m'occupais avant en qualité de responsable d'un service d'urgence interniste d'un hôpital de soins aigus, leurs besoins sont plus aigus. Lorsque j'ai débuté ma nouvelle activité, on m'a souvent demandé (et c'est encore parfois le cas): «Pourquoi as-tu changé? Soigner ces personnes est tellement exigeant, c'est vraiment ce que tu veux? N'est-ce pas trop difficile et déprimant?»

Soigner et surtout accompagner des personnes vulnérables a été un défi, du moins au début, moi qui étais habitué à proposer des solutions et à les appliquer tout de suite. Mais ça ne se passe pas comme ça: au terme de la consultation, certaines patientes et patients quittent la pièce sans même commencer le traitement dont nous avons parlé, d'autres l'acceptent puis l'interrompent, d'autres encore se plaignent auprès des soignants que la ou le médecin peint le diable sur la muraille et qu'elles et ils reviendront plus tard, quand ça ira mal.

Une attention particulière et une réelle écoute sont requises pour déceler de la plus juste manière les besoins et difficultés formulées par ces personnes. Dans certains cas, selon le diagnostic et les limitations qui en découlent, je prescris une hospitalisation, mais les réactions et les comportements varient selon comment je le dis, respectivement comment les patientes et patients le perçoivent: certains s'adressent à un autre membre de notre équipe médicale ou prennent quelques jours de "pause» avant de revenir en nettement moins bonne santé.

Mais dans l'intervalle, elles et ils en parlent presque tous avec leur infirmière ou infirmier de référence. En effet, chez nous, la patientèle peut obtenir ses médicaments auprès du soignant attitré sans forcément devoir consulter le ou la médecin, d'où l'importance zuerich.ch daniel.schroepfer[at] d'une bonne collaboration et communication. Il est ainsi possible d'échanger et obtenir des informations sans passer par la ou le médecin. Que le corps médical se sente trop peu pris au sérieux par la patientèle me semble une erreur: l'enjeu ici est souvent d'arriver à supporter une situation qui n'est agréable pour personne. Nous savons que l'état de santé de nos patientes et patients peut rapidement se dégrader, mais nous avons aussi constaté à maintes reprises à quel point le corps humain est une merveille de la nature.

Parmi ma patientèle, certains suivent un traitement de substitution depuis de nombreuses années, toutefois l'administration du traitement varie de jour en jour. Parfois, il est judicieux de leur donner le médicament juste avant ou après la consultation, afin de les calmer et d'encourager la discussion. L'affirmation selon laquelle patientes et patients seraient ainsi «shootés» n'a pas de sens. Qui songerait à empêcher une personne diabétique en pleine hypoglycémie de prendre un médicament pour stabiliser sa glycémie avant la consultation? De même qu'une personne souffrant d'hypertension hors de contrôle qui se verrait refuser un traitement préalable aurait peu de compréhension et de plaisir à venir consulter. Pour moi, la thérapie de substitution est le traitement de longue durée d'une maladie chronique et n'est liée à aucune tare.

Si nous faisons confiance à nos patientes et patients, elles et ils nous feront également confiance. Ce n'est peut-être pas évident au début, mais le fait qu'elles et ils reviennent régulièrement en consultation en est le signe. Nous suivons certaines personnes depuis 20 ans. Le but du bas seuil n'est pas uniquement d'établir une relation de confiance avec elles, mais de travailler en équipe pour les soutenir et les accompagner. C'est notre patientèle qui nous dit ce dont elle a besoin.

Pour terminer, je dirais que je ne regrette pas un seul instant ce passage à la «médecine sociale» (comme on l'appelait autrefois). Cette activité pose de nombreux défis, mais offre aussi joie et satisfaction et ne peut s'accomplir que grâce à une bonne collaboration. C'est pourquoi je tiens ici à remercier chaleureusement toutes nos équipes. 